\title{
Three-Dimensional Organization of Self-Encapsulating Gluconobacter oxydans Bacterial Cells
}

\author{
Vi Khanh Truong, ${ }^{\dagger}$ Chris M. Bhadra, ${ }^{\dagger}$ Andrew J. Christofferson,,$\oplus \odot$ Irene Yarovsky, ${ }^{*},+$
}

Mohammad Al Kobaisi, ${ }^{\dagger}$ Christopher J. Garvey, ${ }^{,}$Olga N. Ponamoreva," Sergey V. Alferov," Valery A. Alferov, ${ }^{\perp}$ Palalle G. Tharushi Perera, ${ }^{\dagger}$ Duy H. K. Nguyen, ${ }^{\dagger}$ Ričardas Buividas, ${ }^{\dagger}$ Saulius Juodkazis, ${ }^{\dagger}$ Russell J. Crawford, ${ }^{\#}$ and Elena P. Ivanova ${ }^{*},{ }^{\dagger}$

\author{
${ }^{\dagger}$ School of Science, Faculty of Science, Engineering and Technology, Swinburne University of Technology, Hawthorn, Victoria 3122, \\ Australia \\ ${ }^{\ddagger}$ School of Engineering and ${ }^{\#}$ School of Science, RMIT University, GPO Box 2476, Melbourne, Victoria 3001, Australia \\ ${ }^{\S}$ Australian Nuclear Science and Technology Organisation, Locked Bag 2001, Kirrawee DC, Sydney, New South Wales 2232, \\ Australia \\ ${ }$ Biotechnology Department and ${ }^{\perp}$ Chemistry Department, Tula State University, 92 Lenin pr., Tula 300012, Russian Federation
}

\section{Supporting Information}

\begin{abstract}
Self-organized bacteria have been the subject of interest for a number of applications, including the construction of microbial fuel cells. In this paper, we describe the formation of a self-organized, three-dimensional network that is constructed using Gluconobacter oxydans B-1280 cells in a hydrogel consisting of poly(vinyl alcohol) (PVA) with $\mathrm{N}$-vinyl pyrrolidone (VP) as a cross-linker, in which the bacterial cells are organized in a particular side-by-side alignment. We demonstrated that nonmotile G. oxydans cells are able to reorganize themselves, transforming and utilizing PVA-VP polymeric networks through the molecular interactions of bacterial extracellular polysaccharide (EPS) components such as acetan, cellulose, dextran, and levan. Molecular dynamics simulations of the G. oxydans EPS components interacting with the hydrogel polymeric network showed that the solvent-exposed loops of PVA-VP extended and engaged in bacterial selfencapsulation.
\end{abstract}

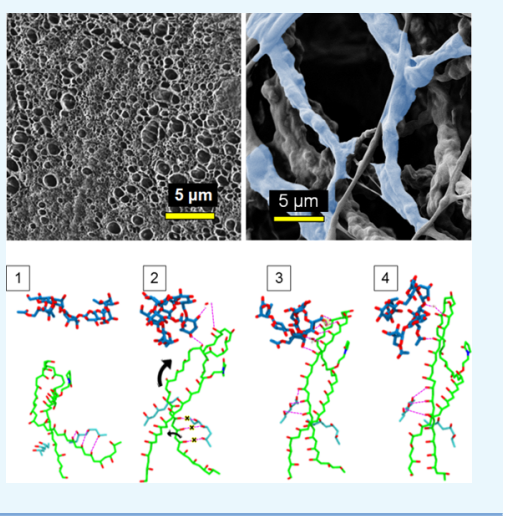

\section{INTRODUCTION}

Despite recent advances and technological innovations in microbial electrochemical technologies, including the bioproduction of biofuel, waste treatment, and biosensing, ${ }^{1-4}$ poor electron transfer between microbes and electrodes is a major concern and a limiting factor for large-scale applications of the technology. $^{2-4}$ In light of this drawback, intensive research has concentrated on understanding the mechanisms responsible for the extracellular electron transfer (EET) process and on methods by which electrocatalytic bacteria communicate. ${ }^{2,4-9}$ It has been discovered that bacteria are able to form "pili-like" nanowires that facilitate an EET process by linking the respiratory chains located on membranes of bacterial cells to adjacent external surfaces, such as oxidized metals in the natural environment, or to engineered electrodes in renewable energy devices. $^{4,6,8,9}$ Bacteria utilize mainly (1) indirect pathways involving redox-active mediators, (2) primary metabolites or other intermediates, or (3) direct modes, involving physical contact in which naturally occurring outer-membrane c-type cytochromes shuttle electrons for the reduction or oxidation of electrodes. ${ }^{10,11}$ Understanding the electron transfer mechanism is important in allowing the optimization of the performance of microbial fuel cells.
Geobacter sulfurreducens bacterial cells have been reported to form pili that serve as biological nanowires that allow the transfer of electrons from bacterial cell surfaces to the surface of Fe(III) oxides. ${ }^{6,7}$ Another metal-reducing bacteria, Shewanella oneidensis MR-1, ${ }^{8,9}$ was observed to form nanowires from the extension of their bacterial outer membrane and periplasm. It was also reported that $S$. oneidensis MR-1 cells that had been treated with cisplatin produced elongated cells, which showed approximately a five-fold improvement in current densities compared to normal, untreated cells. ${ }^{12}$ Furthermore, filamentous bacteria of the family Desulfobulbaceae have been found to conduct electricity over centimeter-long distances, thereby coupling the processes of oxygen reduction at marine sediment surfaces with sulphite oxidation in subsurface layers. ${ }^{5,13}$

Gluconobacter oxydans cells require redox shuttles to assist the transfer of electrons from the active sites of cells to electrodes. ${ }^{1,14-17}$ This Gram-negative, nonmotile bacterium produces periplasmic membrane-bound pyrroloquinoline quinone (PQQ), which contains complex enzymes that allow the

Received: September 1, 2017

Accepted: November 2, 2017

Published: November 20, 2017 
(A)
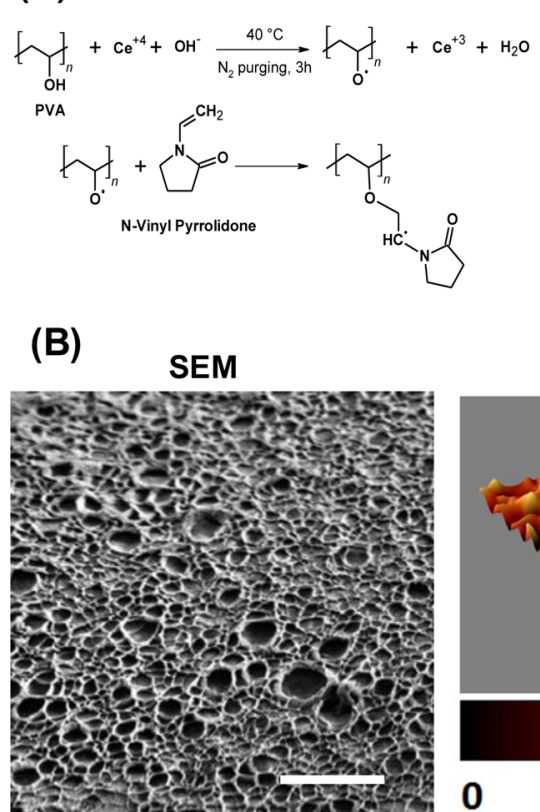

(C)

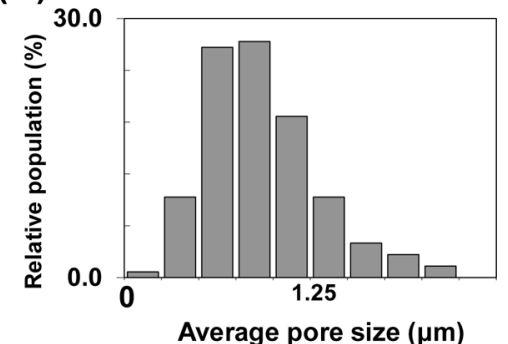

Average pore size $(\mu \mathrm{m})$ Raman

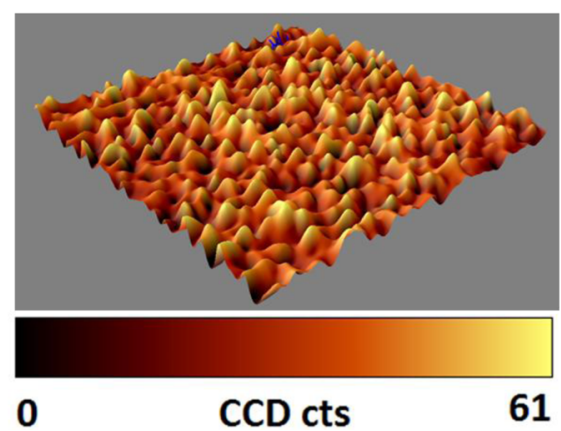

Figure 1. Synthetic pathway and physical characteristics of porous poly(vinyl alcohol) (PVA) of molecular weight $67 \mathrm{kDa}$ cross-linked with VP. (A) Schematic diagram showing the radical polymerization between linear polymeric PVA and VP monomer to form PVA-VP hydrogel. (B) Porous structure of the hydrogel visualized in its freeze-dried and hydrated states using SEM (scale bar $5 \mu \mathrm{m}$ ) and Raman microspectroscopy (scanning areas of $5 \mu \mathrm{m} \times 5 \mu \mathrm{m}$ ). (C) Pore diameter distribution of PVA-VP estimated using ImageJ.

efficient oxidation of a number of substrates. ${ }^{1,14-16,18-20} \mathrm{G}$. oxydans is a promising candidate for the construction of efficient microbial fuel cells because of the periplasmic localization of active sites of PQQ-dependent redox enzymes. ${ }^{1,14-17,20}$ In particular, G. oxydans encapsulated in a confined space showed enhanced levels of electric power generation. ${ }^{14}$ It remains unclear, however, how the G. oxydans cells were encapsulated within polymeric hydrogels. In this paper, we report on the three-dimensional (3D) organization of G. oxydans subsp. industrius B-1280 cells that are encapsulated within the confined environment of a hydrogel system constructed from poly(vinyl alcohol) cross-linked with $\mathrm{N}$ vinyl pyrrolidone (VP). ${ }^{16}$ The proposed mechanism is supported by experimental evidence in addition to molecular dynamics simulation for four exopolysaccharides (acetan, cellulose, dextran, and levan) present on the outer cell wall of the G. oxydans cells. ${ }^{15,18}$ Synthetic and analytical chemistry techniques, including ultra-small-angle neutron scattering (USANS), biological assays and computational modeling were used to demonstrate that G. oxydans cells were engaged in selfencapsulation and were able to construct a network by utilizing the molecular components of the PVA-VP hydrogel. Specifically, we showed that interactions taking place between bacterial extracellular polysaccharide components and surface loops of the PVA-VP hydrogel had the greatest influence over the structure of resultant hydrogel and were able to transform its original three-dimensional structure into a well-organized biological network.

\section{RESULTS AND DISCUSSION}

Three-Dimensional Organization of the PVA-VP Hydrogels. To understand the way in which the G. oxydans cells are encapsulated within the hydrogel, an understanding of the structure of original hydrogel polymeric network is essential. Therefore, the hydrogel was synthesized through the free radical polymerization of linear PVA using VP as a cross-linker. ${ }^{14}$ Ceric ammonium nitrate (CAN) was used to initiate the chain reaction, causing the formation of the free radical species shown in Figure 1A. The free oxygen radicals of PVA were then cross-linked with double bonds present on VP monomers, leading to the formation of a three-dimensional porous polymeric network, as confirmed by scanning electron microscopy (SEM) analysis of freeze-fractured hydrogel samples (Figure 1B). X-ray diffraction was previously used to obtain an insight into the process by which PVA hydrogel porous structures were formed. ${ }^{21}$ The results of this study indicated the presence of entangled swollen amorphous and crystalline domains of PVA that acted as "bulges" within the gel network. Raman microspectroscopic imaging of samples also confirmed the formation of a PVA-VP porous structure within hydrogel films in their hydrated state (Figure $1 \mathrm{~B}$ and Figure S1A,B, Supporting Information). The estimated pore diameter distribution of the resultant hydrogels is presented in Figure 1C. It was found that PVA-VP hydrogels exhibited a pore diameter of $0.92 \pm 0.38 \mu \mathrm{m}$ and a swelling ratio of $410 \%$ (Figure S1C, Supporting Information), most likely due to the low proportion of crystalline segments being present in PVAVP. 22,23

G. oxydans Bacterial Cell Network Formation. The three-dimensional organization of G. oxydans cells in the PVAVP hydrogels was visualized using SEM, confocal laser scanning microscopy (CLSM), and Raman microspectroscopy (RM) (Figure 2). The examination of the freeze-fractured hydrogels containing the bacterial cells showed that the original threedimensional structure of hydrogels had been completely altered. In the hydrogel environment, G. oxydans cells were found to have self-organized into a network (Figure 2A), in contrast to the structure adopted in the absence of PVA-VP (Figure 1). CLSM and Raman microspectroscopy image analyses confirmed that the G. oxydans cells had formed a 

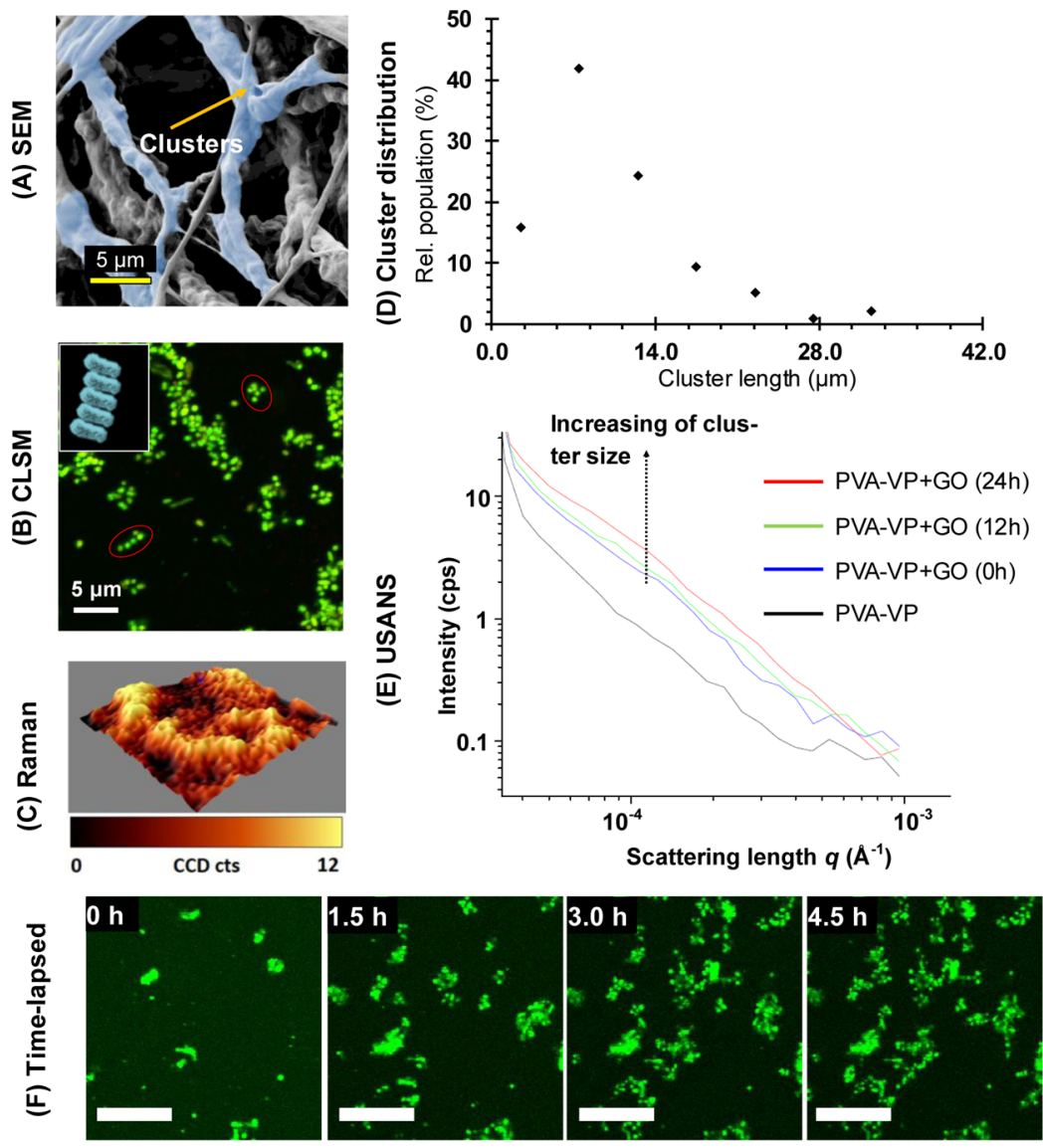

(G) Self-assembly of G. oxydans in polymeric network

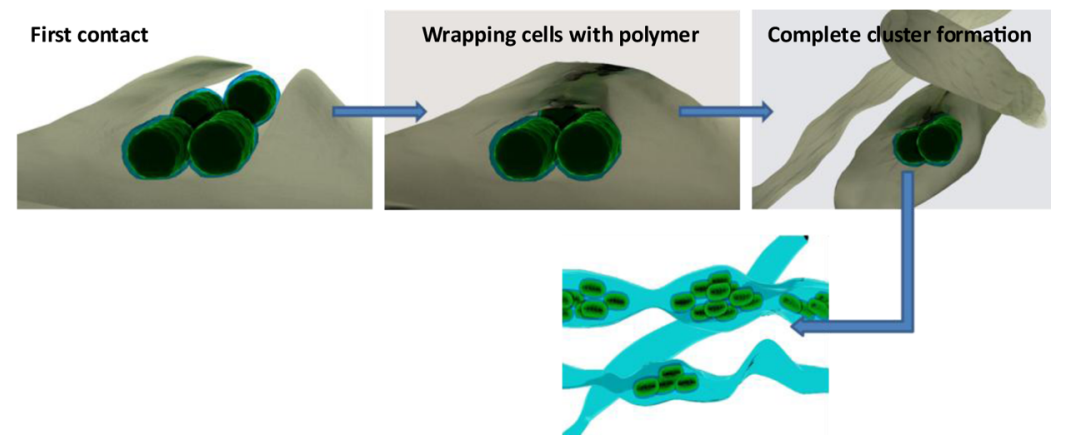

Figure 2. Self-assembly of G. oxydans bacterial cells into the biological network in the PVA-VP hydrogel system. (A) SEM showing the reorganization of G. oxydans cells into clusters wrapped with the polymer (blue highlight indicates the continuous chains of cells). (B) Confocal laser scanning microscopy images, with viable cells stained green with SYTO 9 and nonviable cells stained red with propidium iodide and (C) Raman microspectroscopy images (scanning areas of approximately $5 \times 5 \mu \mathrm{m}^{2}$ ) showing G. oxydans cell clusters. (D) Cluster length distributions of G. oxydans bacterial cells showing that cells formed clusters in $67 \mathrm{kDa}$ PVA-VP hydrogel. The kinetics of self-organization of G. oxydans cells in PVAVP hydrogel was investigated using time-lapsed microscopy (E) CLSM and (F) USANS. (G) Schematic diagram illustrating the re-arrangement of bacteria disrupting the polymeric network.

network in the hydrogel (Figure 2B,C). The average biological cluster lengths were found to be $10.5 \pm 6.4 \mu \mathrm{m}$, as inferred from the ImageJ analysis data (Figure 2D).

The dynamic formation of the network by G. oxydans cells in the PVA-VP hydrogel system was further investigated using time-lapsed CLSM and USANS (Figure 2E,F). Over the period of an hour, short clusters of 4-5 cells were found to have formed (Movie S1, Supporting Information). A further elongation of the short clusters continued after this time for the next $24 \mathrm{~h}$, resulting in the formation of larger self-assembled clusters (Figure 2F, Figure S2A and Movie S2, Supporting
Information). The analysis of the USANS spectra showed that the increased intensity of scattering lengths $(q)$ ranged from 0.5 $\times 10^{-4}$ to $1.0 \times 10^{-4} \AA^{-1}$ over the $24 \mathrm{~h}$ period, indicating that the G. oxydans cell clusters had become larger. Because of the complexity and polydispersity of the globular knot complex structures of PVA-VP together with G. oxydans, further resolving the scattering patterns of USANS was not feasible. However, the change in scattering intensity gradients of the neutron beam clearly indicated the cluster formation by $G$. oxydans cells. A similar approach was previously used to identify 
(A)

(B)
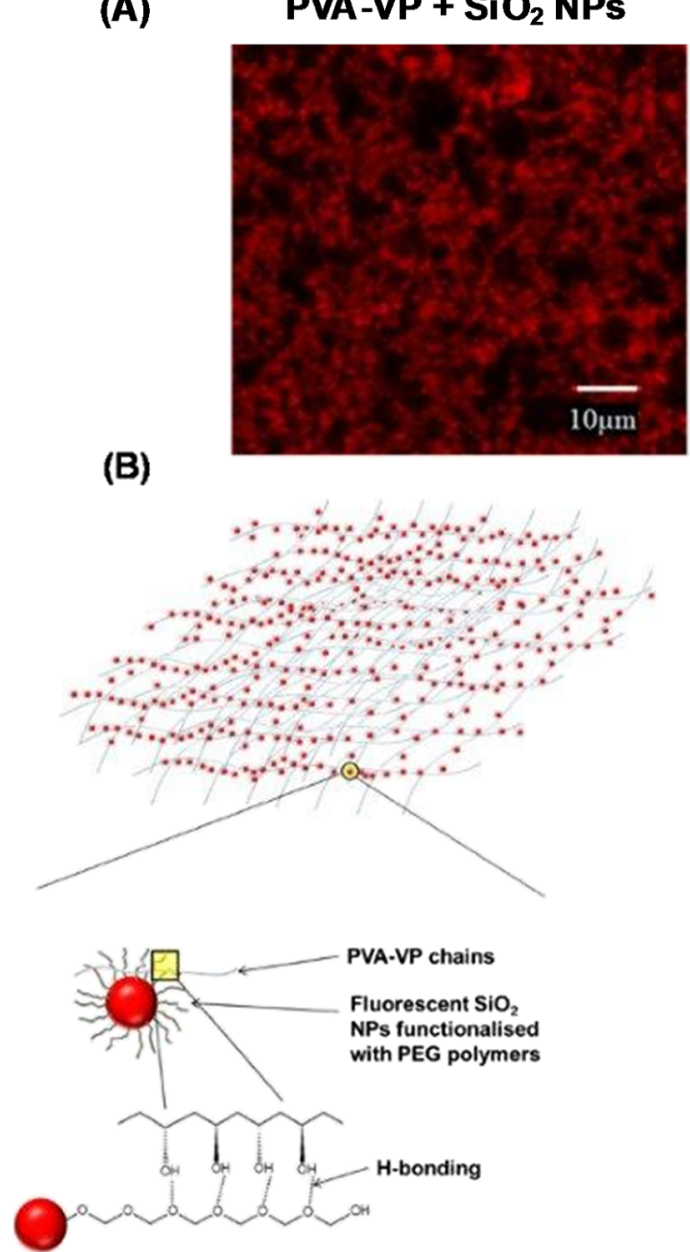

$\mathrm{PVA}-\mathrm{VP}+\mathrm{SiO}_{2} \mathrm{NPs}+\mathrm{G}$. oxydans
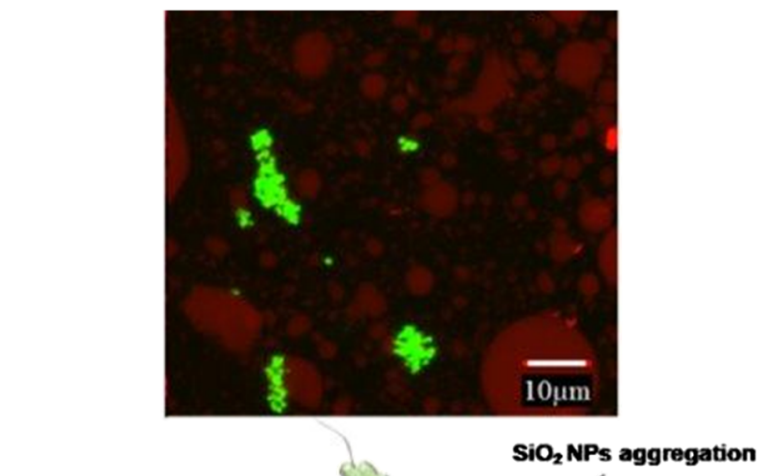

$\mathrm{SiO}_{2} \mathrm{NPS}$ aggregation

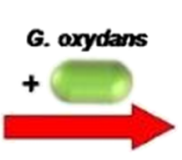

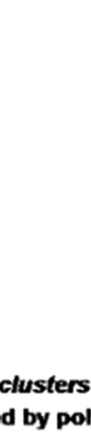

G. oxydans clusters

mer

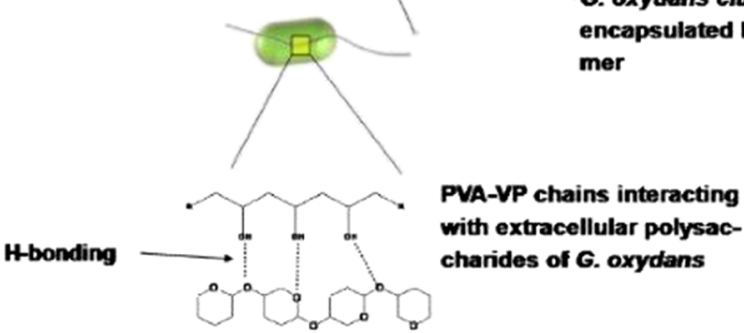

Figure 3. Competitive interactions of bacterial cells and PEG-functionalized $\mathrm{SiO}_{2}$ nanoparticles with the PVA-VP hydrogel. (A) CLSM micrographs showing that G. oxydans bacterial cells (right image, green color) completely disrupted the structure of original hydrogel (left image), fluorescent $\mathrm{SiO}_{2}$ nanoparticles $\left(\mathrm{SiO}_{2} \mathrm{NPs}\right.$ ) (red color) labeled hydroxyl groups of the PVA-VP hydrogel. (B) Schematic diagram of the hypothetical mechanism by which hydrogel polymeric chains and G. oxydans cells interact. Disruption of hydrogen bond formation between PEG-functionalized $\mathrm{SiO}_{2} \mathrm{NPs}$ and PVA-VP hydrogel and hydrogen bond formation between extracellular polysaccharides of G. oxydans cells and PVA-VP.

the formation of the protein clusters, such as those of monoclonal antibodies. ${ }^{24}$

To understand the way in which the G. oxydans cells interact with the PVA-VP hydrogel system, the hydrogel was labeled with polyethylene glycol (PEG)-functionalized fluorescent silica nanoparticles $\left(\mathrm{SiO}_{2} \mathrm{NPs}\right)$ as described elsewhere ${ }^{25,26}$ and in Supporting Information S1.1. The NPs were bound to the $\mathrm{OH}$ groups present on the PVA-VP (Figure 3A). The location of the binding sites of the $\mathrm{SiO}_{2} \mathrm{NPs}$ on the PVA-VP was visualized using CLSM. The CLSM micrographs confirmed the presence of the original porous three-dimensional (3D) organization of the cells, as highlighted by the various positions of the $\mathrm{SiO}_{2} \mathrm{NPs}$ (Figure 3A). After the addition of the G. oxydans cells, however, the $\mathrm{SiO}_{2} \mathrm{NPs}$ were found to have completely dissociated from the PVA-VP hydrogel polymeric chains (Figure 3A). These results suggested that the G. oxydans cells replaced the $\mathrm{SiO}_{2} \mathrm{NPs}$ to occupy the binding sites present in the PVA-VP (Figure 3B). Notably, after over $70 \mathrm{~h}$ of analysis, no statistically significant change was observed in the $\mathrm{pH}$ level of the PVA-VP hydrogel system (Figure S2B, Supporting Information), thus eliminating the possibility that an alteration in the $3 \mathrm{D}$ organization of the hydrogels with bacterial cells could have occurred as a consequence of changes in $\mathrm{pH}$.

The examination of the CLSM micrographs revealed that the G. oxydans cells had secreted an extracellular polymeric material (Figure S1E, Supporting Information) and had adopted a characteristic but partial side-by-side orientation within the clusters being formed (Figure 2B and Figure S1F, Supporting Information). Markedly, when Escherichia coli K12 cells were introduced into the PVA-VP hydrogel, no self-organized clusters were detected (Figure S3, Supporting Information). To the best of our knowledge, a side-by-side alignment in a hydrogel matrix has only been reported for nonbiological, ellipsoidal microparticles. ${ }^{27}$ Examples of self-oriented electroactive cells reported in the literature include the pili or pili-like nanowires formed by $S$. oneidensis ${ }^{8,9,12,28}$ and filamentous bacteria of the family Desulfobulbaceae, which can undergo cable-like alignment. ${ }^{5,13}$ It has also been reported that nonelectroactive bacteria such as Lactobacillus fermentum and Bifidobacterium breve have been able to align themselves in response to the application of an external magnetic field after being coated with maghemite nanoparticles. ${ }^{29}$ In addition to these reports, some artificial systems have been developed to 
mediate the aggregation of nonelectroactive bacteria through the modification of the surfaces of bacterial cells. ${ }^{30,31}$

Biocatalytic Activity of G. oxydans Cell Clusters. The encapsulated G. oxydans cell clusters in PVA-VP hydrogels required only $20-30 \mathrm{~min}$ to generate $90.0 \mathrm{mV} / \mathrm{mg}$, whereas freely suspended bacteria generated lower electricity $(48.0 \mathrm{mV} /$ $\mathrm{mg}$ ) after 60-70 min (Table S1, Supporting Information). The microbial fuel cell containing the encapsulated $G$. oxydans cells was able to maintain the generated electrical potential over a period of 8 days (Figure S4, Supporting Information), whereas the electric potential generated by the suspended cells has been reported to be stable only for a $24 \mathrm{~h}$ period. The conductivity of the bacterial network was estimated to be $0.32 \mathrm{mS} / \mathrm{m}$, which is much higher than that of freely suspended bacteria $(0.11 \mathrm{mS} /$ m) (Table S1, Supporting Information). Cyclic voltammograms (CVs) were recorded using bare graphite electrodes with freely suspended bacteria in the electrochemical cell and at the PVAVP-encapsulated G. oxydans-modified electrode (Figure 4).

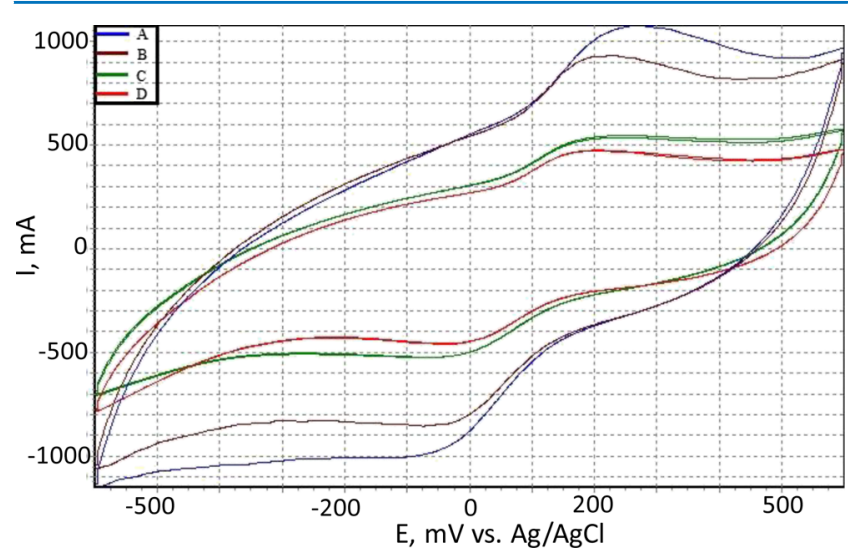

Figure 4. Cyclic voltammograms (CV) of suspended and encapsulated G. oxydans. The cyclic voltammogram (CV) of a (A) bare graphite electrode with free-suspended bacteria $(30 \mathrm{mg})$ and 2,6-DCPIP (0.08 $\mathrm{mM})$ at the electrochemical cell, (B) bare graphite electrode with freesuspended bacteria $(30 \mathrm{mg}), 2,6$-DCPIP $(0.08 \mathrm{mM})$ and glucose $(0.01$ $\mathrm{M})$, (C) PVA-VP/G. oxydans-modified electrode bacteria $(30 \mathrm{mg}$ ) with 2,6-DCPIP $(0.08 \mathrm{mM})$, (D) PVA-VP/G. oxydans-modified electrode $(30 \mathrm{mg})$ with 2,6 -DCPIP $(0.08 \mathrm{mM})$ and glucose $(0.01 \mathrm{M})$, electrolyte: $30 \mathrm{mM}$ phosphate buffer at $\mathrm{pH} 6.0$.

These voltammograms highlighted a significant difference between the two systems. It is suggested that the improved performance of the microbial fuel cells constructed using the encapsulated $G$. oxydans clusters resulted in the increased efficiency in the transfer of extracellular electrons between bacterial clusters.

Molecular Dynamics Simulation of $G$. oxydans Extracellular Polysaccharide Components Interacting with the Hydrogel Polymeric Network. The outer cell wall of the G. oxydans cells is surrounded by an extracellular polysaccharide (EPS) capsule, which typically contains several polysaccharide units, including acetan, cellulose, dextran, and levan. ${ }^{15,18}$ Because the contact between the G. oxydans cells and the PVA-VP hydrogel occurs via the EPS, all-atom model heptamers of the four EPS components were constructed and solvated with explicit water, and their interaction with the model PVA-VP hydrogel was then simulated as a function of time (Figure 5A and Table S2, Supporting Information).

The mesoporous poly(vinyl alcohol) $-N$-vinylpyrrolidone (PVA-VP) hydrogel model was constructed from 5 atactic
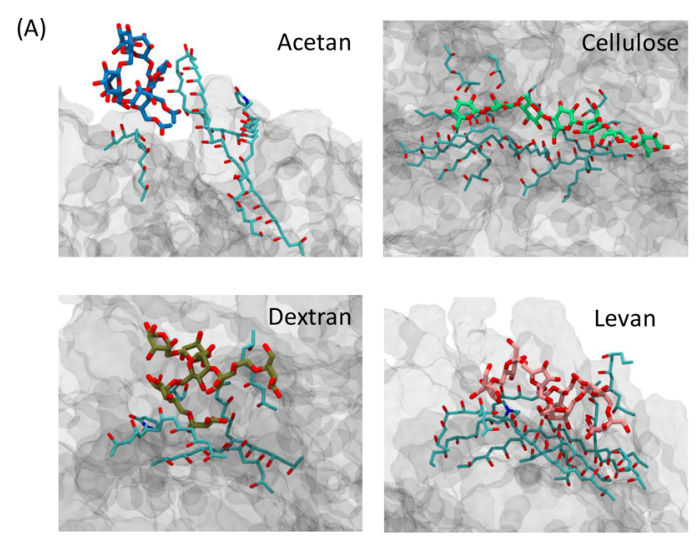

(B)

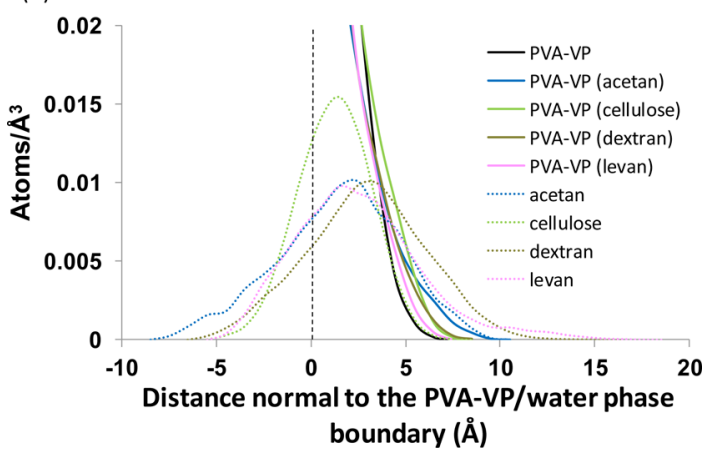

Figure 5. Modification of PVA-VP/water interfaces by different bacterial extracellular polysaccharide components (acetan, cellulose, dextran, and levan). (A) Model structures of four typical polysaccharide components (acetan, cellulose, dextran, and levan) interacting with hydrated PVA-VP polymeric networks (oxygen atoms are colored red, PVA-VP carbon atoms colored cyan, and acetan, cellulose, dextran, and levan carbon atoms colored blue, green, tan, and pink, respectively). (B) Atomic density profile of the PVA$\mathrm{VP} /$ water interface showing the change in atomic density taking place when extracellular polysaccharide components (acetan, cellulose, dextran, and levan) are present compared to PVA-VP alone.

oligomers of 23 monomer units with a molar ratio of 22:1 VA/ $\mathrm{VP}$, which were randomly packed into a confined layer using the Theodorou-Suter algorithm. ${ }^{32,33}$ Following packing, the oligomer termini were attached to the nearest adjacent terminus to create an infinite polymer surface. The system was then solvated with a water volume to reproduce the experimental concentration of polymer in water of $54.9 \mathrm{mg} / \mathrm{mL}$ and simulated using molecular dynamics (MD) for $200 \mathrm{~ns}$ to relax the system. The heptamers of acetan, bacterial cellulose, dextran, and levan were constructed, solvated, and simulated for at least $50 \mathrm{~ns}$ to relax the structures. Following relaxation of the polymer hydrogel and polysaccharide heptamers, each polysaccharide was positioned in a $40 \times 50 \times 70 \AA^{3}$ unit cell containing the solvated polymer with a minimum distance of 10 $\AA$ to the polymer surface to examine the polymer-hydrogel interactions.

Of the four model EPS components, acetan was found to have the greatest influence over the structure of the hydrogel (Figure 5B). Specifically, the solvent-exposed loops of the PVA-VP (shown in light green in Figure 6B and Movie S3, Supporting Information) extended away from the hydrogel surface and bulk phase, and polymer-polymer interactions were replaced by polymer-EPS interactions. The mechanism of the PVA-VP deformation was characterized by the presence of initially metastable direct and water-mediated hydrogen 


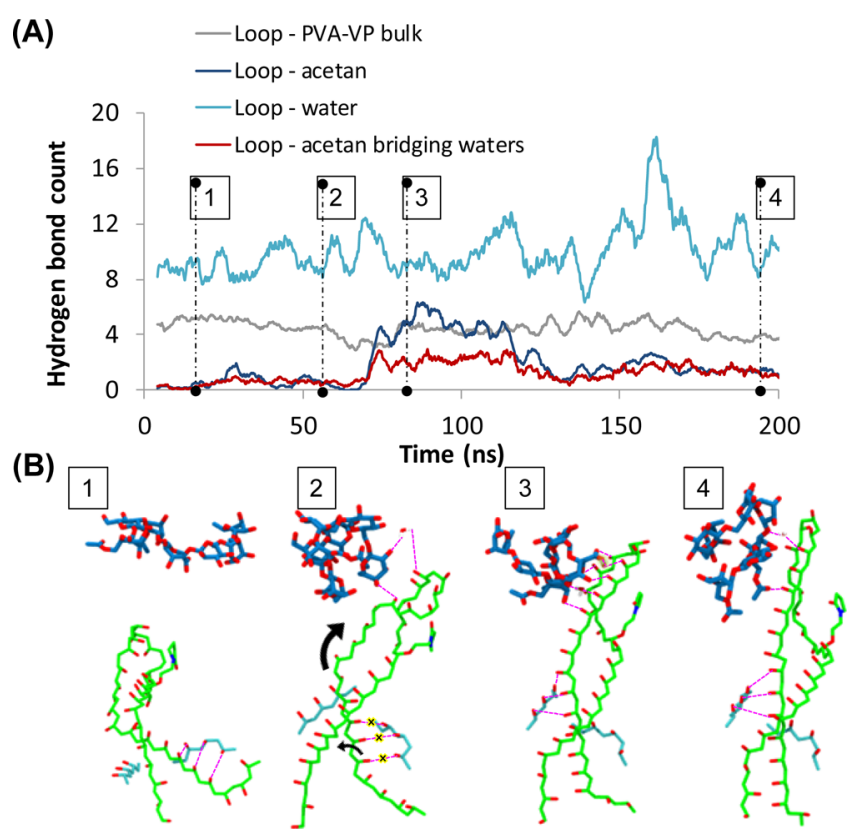

Figure 6. Hydrogen bond counts between a loop of PVA-VP and the PVA-VP bulk, acetan, and water. (A) Hydrogen bond formation between the PVA-VP loops and acetan as a function of time. (B) Corresponding events depicting the hydrogen bond formation taking place between acetan and PVA-VP loops on a molecular scale (oxygen atoms are colored red, PVA-VP carbon atoms are colored green, acetan carbon atoms colored blue, and hydrogen bonding colored purple with dashed lines).

bonds being formed between the acetan and a loop of the PVA-VP (Figure 6). The formation of hydrogen bonds coincided with a breakage of the hydrogen bonds between the exposed polymer loops and the polymer surface, which was accompanied by an increase in the conformational entropy as the loop moved away from the bulk of the polymer. This was followed by additional rearrangements of the loop to maximize the short-range interactions with the acetan molecules. The strong specific interactions taking place between the acetan (and to a lesser extent the other three EPS components) and the loops of the PVA-VP through hydrogen bond formation and van der Waals forces (Figure 6 and Movie S3, Supporting Information) may explain how the bacterial outer layer was able to interact with and modify the structure of the PVA-VP as well as displace the $\mathrm{SiO}_{2}$ NPs from the hydrogel.

Of the four polysaccharides studied, acetan was found to perturb the structure of the PVA-VP hydrogel to the greatest extent, followed by cellulose (Figure 5, Table S2, Supporting Information). Levan exhibited the least effect on the hydrogel. Cellulose remained the most extended polysaccharide throughout the simulations, maintaining a radius of gyration $\left(R_{\mathrm{g}}\right)$ of $10.6 \pm 0.3 \AA$, followed by acetan $(7.4 \pm 1.4 \AA)$. Acetan, however, exhibited the greatest fraction of the contact surface area with the PVA-VP and provided the highest population of polysaccharide-polymer hydrogen bonds of any of the polysaccharides being studied (Table S2, Supporting Information). Interestingly, the interaction energy decomposition analysis indicated that only approximately $25 \%$ of the interaction enthalpy between the acetan and PVA-VP was due to the contribution from electrostatic interactions, whereas the remaining contribution was due to van der Waals interactions. We emphasize that this is a conceptual and representative model of the interactions between the polysaccharides present in the bacterial outer layer and the PVA-VP hydrogel, and all four polysaccharides studied exhibited similar behavior and are capable, in principle, of modifying the polymer structure to some extent. It is likely that all bacterial polysaccharides act cooperatively, causing the withdrawal of individual chains from the polymer bulk, effectively "melting" the polymer network and creating an arrangement favorable to the bacteria.

\section{CONCLUSIONS}

To the best of our knowledge this is the first study that reports the self-organization of $G$. oxydans cells into a biological network by actively utilizing the properties of the PVA-VP hydrogel system in which it is contained. Here, we demonstrated that the self-organization of the G. oxydans cells occurs through the formation of an extended network of bacterial cells aligned in a partial side-by-side configuration to form biological clusters. This particular orientation was most likely adopted to facilitate the efficient transfer of electrons, because it results in an increased interaction contact area. The alignment of the G. oxydans cells in the polymeric hydrogel allowed the microbial fuel cells containing them to generate a greater electric potential than that obtained by suspended $G$. oxydans cells in the absence of the hydrogel. The power generation of the PVA-VP/G. oxydans system was found to be stable for more than 8 days. A remarkable feature of the $G$. oxydans cells is their ability to utilize the PVA-VP hydrogel system to self-encapsulate and self-organize into an extended network via strong specific intermolecular interactions between the polysaccharides present on the exopolysaccharide capsules and the exposed loops of the PVA-VP polymer. This process has the potential to be exploited in the novel design of highly efficient electricity generation cells that utilize components of industrial organic waste.

\section{MATERIALS AND METHODS}

Hydrogel Synthesis. Poly(vinyl alcohol) samples, with molecular weights of $67 \mathrm{kDa}$ (Sigma-Aldrich), were used to form a hydrogel using VP as the cross-linking agent. More details regarding the synthetic procedure for these hydrogels is described in Supporting Information S1.1.

Poly(ethylene oxide)-functionalized fluorescent nanoparticles (of diameter approximately $15 \mathrm{~nm}$ ) were prepared and incorporated into the hydrogel as detailed in Supporting Information S1.1. These were used to characterize the porous cellular microstructure of the hydrogels and to investigate the interactions taking place between the bacteria and the hydrogel. $^{26}$ The swelling behavior of the PVA-VP hydrogels in Milli-Q water was studied and reported in terms of the swelling ratio $(Q)$, where $Q=$ swollen mass/initial dry hydrogel mass. ${ }^{26}$ The detailed procedure is described in Supporting Information S1.1. The synthesis of PVA-VP and nanoparticleimmobilized PVA-VP is described elsewhere ${ }^{14,26}$ and detailed in Supporting Information S1.2.

Encapsulation of G. oxydans and E. coli Bacterial Cells in Hydrogels. G. oxydans subsp. industrius VKM B-1280 was obtained from Russian Culture Collection from Skryabin Institute of Biochemistry and Physiology of Microorganisms, Russian Academy of Science. The G. oxydans cells were grown at $30{ }^{\circ} \mathrm{C}$ in a medium of the following composition: $200.0 \mathrm{~g} / \mathrm{L}$ D-sorbitol (Sigma-Aldrich) and $20.0 \mathrm{~g} / \mathrm{L}$ yeast extract (BD, 
USA). E. coli K12, used for comparative purposes in CLSM studies, was grown in nutrient broth (Oxoid). The bacterial cells were stored in stock solutions prepared with the same medium containing $20 \%$ glycerol. The stock samples $(1 \mathrm{~mL}$ tubes) were stored at $-80{ }^{\circ} \mathrm{C}$. The bacterial cultures were refreshed prior to use on agar plates incubated at $30{ }^{\circ} \mathrm{C}$ for 48 h.

Prior to the encapsulation process, cells were harvested after a $48 \mathrm{~h}$ incubation period and washed twice with $18.0 \mathrm{mM}$ phosphate-citrate buffer ( $\mathrm{pH}$ 6.0). Wet cell pellets were mixed with the hydrogel solutions to obtain a final concentration of 30 $\mathrm{mg} / \mathrm{mL}$. The mixture was stirred for $24 \mathrm{~h}$, after which time the mixture was progressively diluted to obtain the final concentration of $6 \mathrm{mg} / \mathrm{mL}$. Hydrogel films were prepared by casting approximately $300 \mu \mathrm{L}$ of the diluted mixture onto a glass slide and allowing it to dry at $25{ }^{\circ} \mathrm{C}$ for $24 \mathrm{~h}$. The same process of E. coli encapsulation was carried out with $10 \mathrm{mM}$ PBS ( $\mathrm{pH}$ 7.4). A functional analysis of the constructed microbial fuel cell (MFC) with the encapsulated G. oxydans is given in Supporting Information S1.3.

Scanning Electron Microscopy. The synthetic hydrogels prepared with and without the G. oxydans cells were characterized using SEM (ZEISS SUPRA 40VP, Oberkochen, BW, Germany) at $3 \mathrm{kV}$ under 5000×, $10000 \times, 50000 \times$ magnification. Freeze-dried and fractured hydrogel films with and without bacteria were mounted on an L-shaped aluminum holder using a conductive tape and gold-sputtered as previously described. $^{26}$

Raman Spectroscopy. Raman microspectroscopy of the PVA-VP hydrogels with and without $G$. oxydans was mapped using a Raman microspectrometer (WITec) with a $532 \mathrm{~nm}$ laser wavelength $(h \nu=2.33 \mathrm{eV})$. A $100 \times$ objective was used to characterize the hydrogel samples. A grid of 50 spectra $\times 50$ spectra was acquired for a scanning area of $5 \mu \mathrm{m} \times 5 \mu \mathrm{m}$. The integration time for a single spectrum was $2.0 \mathrm{~s}$. Scanning was repeated independently using three different samples. Visualization of the G. oxydans bacterial cell clusters in the PVA-VP was performed without using the freeze-drying preparation steps used for imaging via CLSM and Raman microspectroscopy.

Ultra-Small-Angle Neutron Scattering Spectroscopy. Ultra-small-angle neutron scattering (USANS) was performed using the Kookaburra beamline at the Australian Nuclear Science and Technology Organisation (ANSTO, NSW, Australia) to determine the microstructure of the PVA-VP hydrogels with and without the G. oxydans being present. Two perfect channel-cut crystals were used to measure the broadening of a monochromatic neutron beam with a wavelength of $4.74 \AA$, thus measuring the small-angle scattering from a sample placed between them, allowing a range of momentum transfer quantities to be utilized. The scattering length, $q$, was calculated according to the following equation

$$
q=(4 \pi / \lambda) \times \sin (\theta / 2)
$$

where $q\left(\AA^{-1}\right)$ is the scattering length; $\lambda(\mathrm{nm})$ is the wavelength of incident neutrons; and $\theta$ (rad) is the scattering angle.

A $q$ range of $3 \times 10^{-5}$ to $3 \times 10^{-3} \AA^{-1}$ was used to investigate the self-assembled structure formed between the hydrogel and $G$. oxydans over a $36 \mathrm{~h}$ period. The samples were contained in a $2 \mathrm{~mm}$ path length fused quartz cell with an aperture of $35 \mathrm{~mm}$ $\times 35 \mathrm{~mm}$. Data were reduced using standard procedures that involved the determination of the transmission with respect to an empty cell and normalized in addition to subtraction of the background scattering from the empty sample cell. Incoherent scattering from water, which was approximately $0.8 \mathrm{~cm}^{-1}$, was considered an insignificant contribution to the background spectra over the range being studied.

Confocal Laser Scanning Microscopy. Confocal laser scanning microscopy was used to visualize the proportion of live and dead cells using a LIVE/DEAD BacLight Bacterial Viability Kit, L7012, which contained a mixture of SYTO 9 and propidium iodide fluorescent dyes (Molecular Probes, Invitrogen, Grand Island, NY, USA). SYTO 9 permeated both intact and damaged membranes of the cells, binding to nucleic acids and fluorescing green when excited by a $485 \mathrm{~nm}$ wavelength laser. The propidium iodide entered only the cells that had sustained significant membrane damage, and hence considered to be nonviable, and binds with a higher affinity to the nucleic acids within the cells than does the SYTO 9. Bacterial suspensions were stained according to the method previously reported ${ }^{26}$ and imaged using a FluoView FV10i inverted microscope (Olympus, Tokyo, Japan).

Molecular Dynamics (MD) Model Construction. The published parameters from the condensed-phase optimized molecular potentials for atomistic simulation studies (COMPASS) force field ${ }^{35}$ were applied to all systems, with missing angles and dihedrals taken from the polymer consistent force field (PCFF). ${ }^{36}$ We have used both COMPASS and PCFF previously to model solvated polymer interactions, ${ }^{37}$ and this family of force fields has been shown to be reliable for modeling polymers and interfaces. ${ }^{38-41}$ All simulations were run in the MD code LAMMPS ${ }^{34}$ in the constant pressure and temperature (NPT) ensemble with the Nosé-Hoover thermostat and barostat to maintain a temperature of $298 \mathrm{~K}$ and a pressure of 1 atm and utilized a 1 fs timestep and 10 ps output frequency. Electrostatics beyond a $15.5 \AA$ cutoff were treated with the PPPM summation method with an accuracy of $1 \times 10^{-5} \mathrm{kcal} /$ mol and van der Waals interactions with an atom-based summation utilizing a $15.5 \AA$ cutoff and tail correction. All simulations were run for up to $300 \mathrm{~ns}$, with at least three different initial velocity distributions for each system. Equilibration was defined as the point where the heavy-atom root mean squared deviation (rmsd) reached a steady value with a standard deviation of less than $1 \AA$. All analyses were performed on $10 \mathrm{~ns}$ of equilibrated trajectory. Hydrogen bonds were calculated with a maximum donor-acceptor distance of $2.5 \AA$ and a minimum angle of $120^{\circ}$. Atomic density profiles were created using the VMD Density Profile Tool. ${ }^{42}$

\section{ASSOCIATED CONTENT}

\section{Supporting Information}

The Supporting Information is available free of charge on the ACS Publications website at DOI: 10.1021/acsomega.7b01282.

Supporting materials and methods and results and discussion (PDF)

Self-assembly of G. oxydans in the PVA-VP system over a 1 hour period (AVI)

Time-lapsed CLSM showing the self-assembly of G. oxydans in PVA-VP over a $24 \mathrm{~h}$ period (AVI)

Interactions between acetan and PVA-VP polymer (AVI)

\section{AUTHOR INFORMATION}

\section{Corresponding Authors}

*E-mail: irene.yarovsky@rmit.edu.au (I.Y.). 
*E-mail: eivanova@swin.edu.au (E.P.I.).

ORCID

Andrew J. Christofferson: 0000-0003-0904-6630

Elena P. Ivanova: 0000-0002-5509-8071

Notes

The authors declare no competing financial interest.

\section{ACKNOWLEDGMENTS}

The authors acknowledge partial support from the Marie Curie Actions under EU FP7 Initial Training Network SNAL 608184 http://itn-snal.net/. O.N.P., V.A.A. and S.V.A. would like to acknowledge the financial support from the Ministry of Education and Science of Russian Federation (RFMEF157414X0062). The authors acknowledge the support of the Bragg Institute, Australian Nuclear Science and Technology Organisation (ANSTO), in providing the neutron research facilities used in this work. The authors would also like to thank Dr. Christine Rehm and Dr. Liliana de Campo from ANSTO for their technical support. The authors also thank Dr. Matthew Penna and Patrick Charchar (School of Engineering, RMIT University, Australia) for useful discussions pertaining to this work. A.J.C. and I.Y. acknowledge the generous allocation of the high-performance computational resources from the Australian National Computational Infrastructure (NCI), the Western Australian computational facility (iVEC), the Victorian Partnership for Advanced Computing (VPAC), and the Victorian Life Sciences Computational Initiative (VLSCI).

\section{REFERENCES}

(1) Babkina, E.; Chigrinova, E.; Ponamoreva, O.; Alferov, V.; Reshetilov, A. Bioelectrocatalytic oxidation of glucose by immobilized bacteria Gluconobacter oxydans. Evaluation of water-insoluble mediator efficiency. Electroanalysis 2006, 18, 2023-2029.

(2) Logan, B. E. Exoelectrogenic bacteria that power microbial fuel cells. Nat. Rev. Microbiol. 2009, 7, 375-381.

(3) ElMekawy, A.; Hegab, H. M.; Dominguez-Benetton, X.; Pant, D. Internal resistance of microfluidic microbial fuel cell: Challenges and potential opportunities. Bioresour. Technol. 2013, 142, 672-682.

(4) Huang, L.; Regan, J. M.; Quan, X. Electron transfer mechanisms, new applications, and performance of biocathode microbial fuel cells. Bioresour. Technol. 2011, 102, 316-323.

(5) Marzocchi, U.; Trojan, D.; Larsen, S.; Meyer, R. L.; Revsbech, N. P.; Schramm, A.; Nielsen, L. P.; Risgaard-Petersen, N. Electric coupling between distant nitrate reduction and sulfide oxidation in marine sediment. ISME J. 2014, 8, 1682-1690.

(6) Reguera, G.; McCarthy, K. D.; Mehta, T.; Nicoll, J. S.; Tuominen, M. T.; Lovley, D. R. Extracellular electron transfer via microbial nanowires. Nature 2005, 435, 1098-1101.

(7) Malvankar, N. S.; Vargas, M.; Nevin, K. P.; Franks, A. E.; Leang, C.; Kim, B.-C.; Inoue, K.; Mester, T.; Covalla, S. F.; Johnson, J. P.; et al. Tunable metallic-like conductivity in microbial nanowire networks. Nat. Nanotechnol. 2011, 6, 573-579.

(8) Pirbadian, S.; Barchinger, S. E.; Leung, K. M.; Byun, H. S.; Jangir, Y.; Bouhenni, R. A.; Reed, S. B.; Romine, M. F.; Saffarini, D. A.; Shi, L.; et al. Shewanella oneidensis MR-1 nanowires are outer membrane and periplasmic extensions of the extracellular electron transport components. Proc. Natl. Acad. Sci. U.S.A. 2014, 111, 12883-12888.

(9) Gorby, Y. A.; Yanina, S.; McLean, J. S.; Rosso, K. M.; Moyles, D.; Dohnalkova, A.; Beveridge, T. J.; Chang, I. S.; Kim, B. H.; Kim, K. S.; et al. Electrically conductive bacterial nanowires produced by Shewanella oneidensis strain MR-1 and other microorganisms. Proc. Natl. Acad. Sci. U.S.A. 2006, 103, 11358-11363.

(10) Patil, S. A.; Hägerhäll, C.; Gorton, L. Electron transfer mechanisms between microorganisms and electrodes in bioelectrochemical systems. Bioanal. Rev. 2012, 4, 159-192.
(11) Hernandez, M. E.; Newman, D. K. Extracellular electron transfer. Cell. Mol. Life Sci. 2001, 58, 1562-1571.

(12) Patil, S. A.; Górecki, K.; Hägerhäll, C.; Gorton, L. Cisplatininduced elongation of Shewanella oneidensis MR-1 cells improves microbe-electrode interactions for use in microbial fuel cells. Energy Environ. Sci. 2013, 6, 2626-2630.

(13) Pfeffer, C.; Larsen, S.; Song, J.; Dong, M.; Besenbacher, F.; Meyer, R. L.; Kjeldsen, K. U.; Schreiber, L.; Gorby, Y. A.; El-Naggar, M. Y.; et al. Filamentous bacteria transport electrons over centimetre distances. Nature 2012, 491, 218-221.

(14) Alferov, S. V.; Minaicheva, P. R.; Arlyapov, V. A.; Asulyan, L. D.; Alferov, V. A.; Ponamoreva, O. N.; Reshetilov, A. N. Bioanode for a microbial fuel cell based on Gluconobacter oxydans immobilized into a polymer matrix. Appl. Biochem. Microbiol. 2014, 50, 637-643.

(15) Deppenmeier, U.; Hoffmeister, M.; Prust, C. Biochemistry and biotechnological applications of Gluconobacter strains. Appl. Microbiol. Biotechnol. 2002, 60, 233-242.

(16) Reshetilov, A.; Alferov, S.; Tomashevskaya, L.; Ponamoreva, O. Testing of bacteria Gluconobacter oxydans and electron transport mediators composition for application in biofuel cell. Electroanalysis 2006, 18, 2030-2034.

(17) Vostiar, I.; Ferapontova, E. E.; Gorton, L. Electrical "wiring" of viable Gluconobacter oxydans cells with a flexible osmium-redox polyelectrolyte. Electrochem. Commun. 2004, 6, 621-626.

(18) De Muynck, C.; Pereira, C. S. S.; Naessens, M.; Parmentier, S.; Soetaert, W.; Vandamme, E. J. The genus Gluconobacter oxydans: Comprehensive overview of biochemistry and biotechnological applications. Crit. Rev. Biotechnol. 2007, 27, 147-171.

(19) Prust, C.; Hoffmeister, M.; Liesegang, H.; Wiezer, A.; Fricke, W. F.; Ehrenreich, A.; Gottschalk, G.; Deppenmeier, U. Complete genome sequence of the acetic acid bacterium Gluconobacter oxydans. Nat. Biotechnol. 2005, 23, 195-200.

(20) Treu, B. L.; Minteer, S. D. Isolation and purification of PQQdependent lactate dehydrogenase from Gluconobacter and use for direct electron transfer at carbon and gold electrodes. Bioelectrochemistry 2008, 74, 73-77.

(21) Ricciardi, R.; Auriemma, F.; De Rosa, C.; Lauprêtre, F. X-ray diffraction analysis of poly(vinyl alcohol) hydrogels, obtained by freezing and thawing techniques. Macromolecules 2004, 37, 19211927.

(22) Rubinstein, M.; Colby, R. H.; Dobrynin, A. V.; Joanny, J.-F. Elastic modulus and equilibrium swelling of polyelectrolyte gels. Macromolecules 1996, 29, 398-406.

(23) Lee, K. Y.; Rowley, J. A.; Eiselt, P.; Moy, E. M.; Bouhadir, K. H.; Mooney, D. J. Controlling mechanical and swelling properties of alginate hydrogels independently by cross-linker type and cross-linking density. Macromolecules 2000, 33, 4291-4294.

(24) Yearley, E. J.; Godfrin, P. D.; Perevozchikova, T.; Zhang, H.; Falus, P.; Porcar, L.; Nagao, M.; Curtis, J. E.; Gawande, P.; Taing, R.; et al. Observation of small cluster formation in concentrated monoclonal antibody solutions and its implications to solution viscosity. Biophys. J. 2014, 106, 1763-1770.

(25) Wang, X.-d.; Stolwijk, J. A.; Lang, T.; Sperber, M.; Meier, R. J.; Wegener, J.; Wolfbeis, O. S. Ultra-small, highly stable, and sensitive dual nanosensors for imaging intracellular oxygen and $\mathrm{pH}$ in cytosol. J. Am. Chem. Soc. 2012, 134, 17011-17014.

(26) Truong, V. K.; Mainwaring, D. E.; Murugaraj, P.; Nguyen, D. H. K.; Ivanova, E. P. Impact of confining 3-D polymer networks on dynamics of bacterial ingress and self-organisation. J. Mater. Chem. B 2015, 3, 8704-8710.

(27) Botto, L.; Yao, L.; Leheny, R. L.; Stebe, K. J. Capillary bond between rod-like particles and the micromechanics of particle-laden interfaces. Soft Matter 2012, 8, 4971-4979.

(28) Babauta, J. T.; Nguyen, H. D.; Beyenal, H. Redox and pH microenvironments within Shewanella oneidensis MR-1 biofilms reveal an electron transfer mechanism. Environ. Sci. Technol. 2011, 45, 66546660. 
(29) Martín, M.; Carmona, F.; Cuesta, R.; Rondón, D.; Gálvez, N.; Domínguez-Vera, J. M. Artificial magnetic bacteria: Living magnets at room temperature. Adv. Funct. Mater. 2014, 24, 3489-3493.

(30) Drachuk, I.; Calabrese, R.; Harbaugh, S.; Kelley-Loughnane, N.; Kaplan, D. L.; Stone, M.; Tsukruk, V. V. Silk macromolecules with amino acid-poly(ethylene glycol) grafts for controlling layer-by-layer encapsulation and aggregation of recombinant bacterial cells. ACS Nano 2015, 9, 1219-1235.

(31) Sankaran, S.; Kiren, M. C.; Jonkheijm, P. Incorporating bacteria as a living component in supramolecular self-assembled monolayers through dynamic nanoscale interactions. ACS Nano 2015, 9, 35793586.

(32) Theodorou, D. N.; Suter, U. W. Detailed molecular structure of a vinyl polymer glass. Macromolecules 1985, 18, 1467-1478.

(33) Theodorou, D. N.; Suter, U. W. Atomistic modeling of mechanical properties of polymeric glasses. Macromolecules 1986, 19, $139-154$.

(34) Plimpton, S. Fast parallel algorithms for short-range molecular dynamics. J. Comput. Phys. 1995, 117, 1-19.

(35) Sun, H. Compass: An ab initio force-field optimized for condensed-phase applications-overview with details on alkane and benzene compounds. J. Phys. Chem. B 1998, 102, 7338-7364.

(36) Sun, H.; Mumby, S. J.; Maple, J. R.; Hagler, A. T. An ab initio CFF93 all-atom force field for polycarbonates. J. Am. Chem. Soc. 1994, 116, 2978-2987.

(37) Yiapanis, G.; Christofferson, A. J.; Plazzer, M.; Weir, M. P.; Prime, E. L.; Qiao, G. G.; Solomon, D. H.; Yarovsky, I. Molecular mechanism of stabilization of thin films for improved water evaporation protection. Langmuir 2013, 29, 14451-14459.

(38) Chen, X. P.; Liang, Q. H.; Jiang, J. K.; Wong, C. K. Y.; Leung, S. Y. Y.; Ye, H. Y.; Yang, D. G.; Ren, T. L. Functionalization-induced changes in the structural and physical properties of amorphous polyaniline: A first-principles and molecular dynamics study. Sci. Rep. 2016, 6, 20621.

(39) Jiang, S.; Jones, J. T. A.; Hasell, T.; Blythe, C. E.; Adams, D. J.; Trewin, A.; Cooper, A. I. Porous organic molecular solids by dynamic covalent scrambling. Nat. Commun. 2011, 2, 207.

(40) Ou, X.; Han, Q.; Dai, H.-H.; Wang, J. Molecular dynamic simulations of the water absorbency of hydrogels. J. Mol. Model. 2015, 21, 231.

(41) Li, X.; Murthy, N. S.; Becker, M. L.; Latour, R. A. Multiscale approach for the construction of equilibrated all-atom models of a poly(ethylene glycol)-based hydrogel. Biointerphases 2016, 11, 021002.

(42) Giorgino, T. Computing 1-D atomic densities in macromolecular simulations: The density profile tool for VMD. Comput. Phys. Commun. 2014, 185, 317-322. 